\title{
Intervalos de referência do pico de velocidade sistólica da artéria cerebral média fetal na população brasileira*
}

\author{
Nomogram of fetal middle cerebral artery peak systolic velocity in a Brazilian population
}

Luciano Marcondes Machado Nardozza ${ }^{1}$, Edward Araujo Junior ${ }^{2}$, Christiane Simioni $^{3}$, Luiz Camano ${ }^{4}$, Antonio Fernandes Moron ${ }^{5}$

Resumo OBJETIVO: Determinar uma curva de referência baseada em múltiplos da mediana para o pico de velocidade sistólica da artéria cerebral média fetal. MATERIAIS E MÉTODOS: Realizou-se estudo de corte transversal com 143 gestantes normais entre 23 e 35 semanas. Realizou-se varredura bidimensional em corte axial do crânio fetal, incluindo os tálamos e o septo pelúcido, e em seguida acionou-se o modo color Doppler, visualizando-se a artéria cerebral média. 0 Doppler pulsátil foi disposto próximo à origem deste vaso, utilizando-se ângulo de insonação de menos de $20^{\circ}$. Para avaliar a correlação do pico de velocidade sistólica da artéria cerebral média com a idade gestacional, utilizou-se o coeficiente de correlação de Person $(r)$. Por meio de modelos de regressão, construiu-se uma tabela de múltiplos da mediana para o pico de velocidade sistólica da artéria cerebral média em cada idade gestacional avaliada, e adicionalmente determinaram-se valores de referência para essa variável. RESULTADOS: Observou-se forte correlação entre o pico de velocidade sistólica da artéria cerebral média e a idade gestacional $(r=0,70 ; p=0,001)$. Determinaram-se valores do pico de velocidade sistólica da artéria cerebral média para os seguintes múltiplos da mediana: 1,0; 1,29; 1,5 ; 1,55. Determinaram-se os percentis 2,5 e 97,5 para o pico de velocidade sistólica da artéria cerebral média, variando de $24,33 \mathrm{~cm}^{2} / \mathrm{s}$ a $78,36 \mathrm{~cm}^{2} / \mathrm{s}$. CONCLUSÃO: Um nomograma do pico de velocidade sistólica da artéria cerebral média fetal foi determinado.

Unitermos: Artéria cerebral média; Doppler; Ultra-sonografia; Nomograma; Feto.

Abstract OBJECTIVE: To determine a reference curve for the peak systolic velocity of fetal middle cerebral artery. MATERIALS AND METHODS: The authors developed a cross-sectional study with 143 healthy pregnant women between the 23rd and 35th gestational weeks. A bidimensional axial scan of the fetal skull was performed, including the thalami and pellucid septum. Subsequently, the middle cerebral artery was visualized with the color Doppler mode. The pulsed-wave Doppler transducer was positioned over the origin of this vessel, at $<20^{\circ}$ insonation angle. The correlation between the peak systolic velocity of the middle cerebral artery and gestational age was evaluated by means of the Pearson's correlation coefficient $(r)$. Regression models were utilized in the construction of a table with multiples of the medians of the middle cerebral artery peak systolic velocity for each gestational age. Additionally, reference values for this variable were determined. RESULTS: A strong correlation was observed between the middle cerebral artery peak systolic velocity and gestational age $(r=0.70 ; p<0.001)$. Values of middle cerebral artery peak systolic velocity were calculated for the following multiples of the medians: $1.0,1.29,1.5,1.55$. The 2.5 th and 97.5 th percentiles were determined for the middle cerebral artery peak systolic velocity ranging between $24.33 \mathrm{~cm}^{2} / \mathrm{s}$ and 78.36 $\mathrm{cm}^{2} / \mathrm{s}$. CONCLUSION: A nomogram for the fetal middle cerebral artery peak systolic velocity during the second half of pregnancy was generated.

Keywords: Middle cerebral artery; Doppler; Ultrasonography; Nomogram; Fetus.

Nardozza LMM, Araujo Jr E, Simioni C, Camano L, Moron AF. Intervalos de referência do pico de velocidade sistólica da artéria cerebral média fetal na população brasileira. Radiol Bras. 2008;41(6):385-389.

\footnotetext{
* Trabalho realizado na Disciplina de Medicina Fetal do Departamento de Obstetrícia da Universidade Federal de São PauIo/Escola Paulista de Medicina (Unifesp/EPM), São Paulo, SP, Brasil.

1. Doutor, Professor Adjunto da Universidade Federal de São Paulo/Escola Paulista de Medicina (Unifesp/EPM), São Paulo, SP, Brasil.

2. Doutor, Médico da Disciplina de Medicina Fetal do Departamento de Obstetrícia da Universidade Federal de São Paulo/ Escola Paulista de Medicina (Unifesp/EPM), São Paulo, SP, Brasil.

3. Especialização em Medicina Fetal, Mestranda da Universidade Federal de São Paulo/Escola Paulista de Medicina (Unifesp/ EPM), São Paulo, SP, Brasil.
}

4. Professor Titular da Universidade Federal de São Paulo/ Escola Paulista de Medicina (Unifesp/EPM), São Paulo, SP, Brasil.

5. Professor Titular, Chefe do Departamento de Obstetrícia da Universidade Federal de São Paulo/Escola Paulista de Medicina (Unifesp/EPM), São Paulo, SP, Brasil.

Endereço para correspondência: Dr. Luciano Marcondes Machado Nardozza. Avenida Lopes de Azevedo, 888, Cidade Jardim. São Paulo, SP, Brasil, 05603-001. E-mail: lunardozza@ uol.com.br

Recebido para publicação em 18/3/2008. Aceito, após revisão, em 30/5/2008.

\section{INTRODUÇÃO}

A aloimunização pelas células vermelhas do sangue é caracterizada por ser distúrbio imunológico conseqüente a incompatibilidade sanguínea materno-fetal, que determina doença hemolítica no feto e no recém-nascido. Há destruição excessivamente rápida das hemácias, com conseqüente anemia, hiperbilirrubinemia e grave 
edema generalizado. É causada por anticorpos específicos produzidos pela mãe, que passam para a circulação fetal durante a gravidez.

Após a descoberta da fisiopatologia, iniciaram-se pesquisas em técnicas de diagnóstico capazes de quantificar o grau de anemia, de forma a diminuir as taxas de morbidade e mortalidade perinatais. Em 1961, Liley ${ }^{(\mathbf{1})}$ descreveu o método de leitura espectrofotométrica do líquido amniótico para determinar indiretamente a concentração de bilirrubina no líquido amniótico e avaliar o estado hemolítico, sendo o líquido amniótico obtido por meio deamniocentese. Todavia, a amniocentese está associada a complicações como ruptura prematura de membranas, parto pré-termo, hemorragia vaginal, infecção e perda gestacional em $0,5 \%$.

No início da década de 80 , com a descoberta da cordocentese guiada por ultrasom de alta resolução, esta se tornou o método padrão-ouro para avaliar o grau de anemia nos casos de aloimunização pelas células vermelhas do sangue ${ }^{(3)}$. Entretanto, a cordocentese está associada a piora da aloimunização materna e perda fetal ${ }^{(4)}$.

No final da década de 80 , surgiram estudos com o uso do Doppler acoplado à ultra-sonografia que tentaram avaliar parâmetros hemodinâmicos fetais que pudessem demonstrar o grau de anemia fetal ${ }^{(5,6)}$. O principal objetivo desses estudos era determinar um método acurado que não apresentasse os riscos dos procedimentos invasivos.

Em meados da década de 90, Mari et al. ${ }^{(7)}$ demonstraram que o pico de velocidade sistólica na artéria cerebral média (PVS-ACM) apresentava-se aumentado. Além disso, demonstraram que este vaso é facilmente visualizado com um ângulo diferente de 0 grau e que sua medida apresenta baixa variabilidade intra- e interobservador ${ }^{(6)}$. Esses mesmos autores determinaram a primeira curva de referência para o PVS-ACM utilizando-se de múltiplos da mediana ${ }^{(8)}$.

O objetivo deste estudo é determinar uma curva de referência para o PVS-ACM fetal, utilizando múltiplos da mediana, e também determinar percentis para este parâmetro em gestantes normais da população brasileira.

\section{MATERIAIS E MÉTODOS}

Realizou-se estudo de corte transversal, no período de agosto de 2006 a agosto de 2007, com 153 gestantes atendidas no Setor de Medicina Fetal do Departamento de Obstetrícia da Universidade Federal de São Paulo/Escola Paulista de Medicina (Unifesp/EPM), São Paulo, SP. Deste total, dez gestantes foram excluídas da análise final por causa das seguintes condições: cinco apresentaram teste de Coombs indireto positivo, três não apresentaram condições técnicas favoráveis à realização do exame e outras duas apresentavam doenças crônicas (uma com cardiopatia e uma com lúpus eritematoso sistêmico). O estudo foi aprovado pelo Comitê de Ética em Pesquisa da Unifesp/EPM, sendo que as pacientes que concordaram na participação voluntária assinaram termo de consentimento.

Foram avaliadas 143 gestantes normais portando fetos únicos, sem malformações diagnosticadas no período pré-natal ou confirmadas no período pós-natal, com idade gestacional entre 24 e 34 semanas completas. Para a determinação da idade gestacional, utilizou-se como parâmetro a data da última menstruação (DUM) em mulheres com DUM regular. Nos casos de DUM incerta, utilizou-se datação baseada em ultra-sonografia realizada até 20 semanas de gestação.

Os limites da idade gestacional foram estabelecidos com base nos critérios de viabilidade fetal, constituindo-se este um período crítico para o nascimento de fetos prematuros que potencialmente se beneficiariam de uma avaliação adequada do fluxo vascular.

Todos os exames foram realizados por apenas um examinador (autor principal), sendo as pacientes avaliadas uma única vez (corte transversal). Todos os exames foram realizados em aparelho modelo SA-8000 Live (Medison; Seoul, Korea), equipado com transdutor convexo volumétrico. A análise dos volumes foi realizada utilizando-se um software chamado SonoView Pro (Medison; Seoul, Korea).

Foram considerados os seguintes ajustes do modo Doppler para a obtenção do PVS-ACM: média de quadros (10), frequiência de penetração (baixa), enhance (1), reject (8), ganho (50), frame average (2), sensibilidade (15), densidade (acionado), balanço (16), scala (1,5 kHz) e filtro (1).

A avaliação Doppler para obtenção do PVS-ACM baseou-se na técnica descrita por Mari et al. ${ }^{(8)}$. O modo color Doppler foi acionado e um corte transverso do crânio fetal ao nível da base do cérebro foi realizado. O polígono de Willis foi visualizado e a ACM mais próxima ao transdutor foi selecionada para o cálculo do PVS. A artéria foi insonada próximo à sua origem na artéria carótida interna, pelo fato de o pico sistólico diminuir com a distância a partir do ponto de origem desse vaso. $\mathrm{O}$ ângulo entre o feixe do ultra-som e o fluxo sanguíneo foi padronizado em $20^{\circ}$. Em seguida, acionou-se o modo Doppler pulsátil, com determinação do PVS-ACM. Foram obtidas três mensurações do PVS, utilizandose a maior medida obtida.

Todos os dados coletados foram armazenados em planilha do programa Excel (Microsoft; USA). A análise estatística foi realizada pelo software SPSS for Windows versão 15.0 (SPSS Inc.; Chicago, USA). Para se avaliar a correlação entre o PVSACM e a idade gestacional, utilizaram-se o coeficiente de correlação de Pearson $(r)$ e diagramas de dispersão, e foram criados modelos de regressão polinomial. Com base nesses modelos de regressão, foi construída para cada idade gestacional (semanas completas) uma tabela de valores baseados em múltiplos da mediana, segundo método utilizado por Mari et al. ${ }^{(8)}$. Adicionalmente, foram construídos valores de referência (percentis), de acordo com o proposto por Royston e Wright ${ }^{(9)}$.

\section{RESULTADOS}

As gestações foram acompanhadas até o nascimento. A idade gestacional média foi de 37 semanas e 2 dias ( 28 semanas e 4 dias a 41 semanas) e o peso ao nascimento, de $2.877 \mathrm{~g}(1.400 \mathrm{a} 4.100 \mathrm{~g})$.

A média de dosagem de hemoglobina nos recém-nascidos foi de 12,48 g/dl (10,1 a $16,1 \mathrm{~g} / \mathrm{dl}$ ) e o hematócrito médio foi de $48,94 \%$ (37,1\% a 62,0\%). Nenhum nascituro necessitou de transfusão no berçário e não houve natimortos ou óbitos neonatais precoces.

Observou-se uma forte correlação entre a idade gestacional e o PVS-ACM $(r=$ 
$0,70 ; p<0,001)$. O diagrama de dispersão entre o PVS-ACM e a idade gestacional mostrou que a relação entre ambos não é linear. O gráfico sugere um ajuste de regressão exponencial ou polinomial entre esses dados (Figura 1). A equação que melhor descreve a relação entre o PVS-ACM e a idade gestacional sugere um ajuste de regressão cúbica:

PVS esperado $=44,5998-0,0874(\mathrm{IG})^{2}+$ $+0,0029(\mathrm{IG})^{3}$.

A partir deste ajuste de regressão, foi construída uma tabela com valores baseados em múltiplos da mediana, de acordo com o método utilizado por Mari et al. ${ }^{(\mathbf{8})}$. Segundo esses autores, os múltiplos da mediana são calculados através da razão entre o valor medido e o valor esperado para a idade gestacional determinado pelo modelo de regressão ajustado. Dessa forma, dado o resultado da paciente, o múltiplo da mediana corresponde ao percentual do valor esperado (valores maiores que 1 indicam que o resultado observado está acima do esperado para aquela idade gestacional). Logo, os valores da coluna "mediana" correspondem aos valores esperados, e as demais correspondem a $29 \%, 50 \%$ e $55 \%$ acima do valor esperado (Tabela 1 ).

A determinação de valores de referência do PVS-ACM baseou-se nos critérios

Tabela 1 Valores do pico de velocidade sistólica na artéria cerebral média $(\mathrm{cm} / \mathrm{s})$ baseados em múltiplos da mediana entre 23 e 35 semanas de gestação.

\begin{tabular}{ccccc}
\hline & \multicolumn{4}{c}{ Múltiplos da mediana para PVS-ACM } \\
\cline { 2 - 5 } IG (sem.) & 1,0 & 1,29 & 1,50 & 1,55 \\
\hline 23 & 35,44 & 45,72 & 53,16 & 54,93 \\
24 & 35,48 & 45,77 & 53,22 & 55,00 \\
25 & 35,81 & 46,20 & 53,72 & 55,51 \\
26 & 36,45 & 47,03 & 54,68 & 56,50 \\
27 & 37,43 & 48,29 & 56,15 & 58,02 \\
28 & 38,77 & 50,01 & 58,15 & 60,09 \\
29 & 40,49 & 52,23 & 60,73 & 62,75 \\
30 & 42,61 & 54,97 & 63,91 & 66,04 \\
31 & 45,16 & 58,26 & 67,74 & 70,00 \\
32 & 48,17 & 62,13 & 72,25 & 74,66 \\
33 & 51,65 & 66,62 & 77,47 & 80,05 \\
34 & 55,63 & 71,76 & 83,44 & 86,22 \\
35 & 60,13 & 77,56 & 90,19 & 93,20 \\
\hline
\end{tabular}

IG (sem.), idade gestacional em semanas; PVS-ACM, pico de velocidade sistólica na artéria cerebral média.

propostos por Royston e Wright ${ }^{(\mathbf{9})}$. Os valores são baseados em modelos de regressão paras as médias e desvios-padrão (DP). Para o ajuste do DP, utilizou-se um modelo quadrático:

$$
\begin{aligned}
{[\mathrm{DP}=} & 43,1872-2,8425(\mathrm{IG})+ \\
& \left.+0,0530(\mathrm{IG})^{2}\right] .
\end{aligned}
$$

A partir da média e DP ajustados para cada idade gestacional estudada, calcularam-se os percentis pela seguinte fórmula:

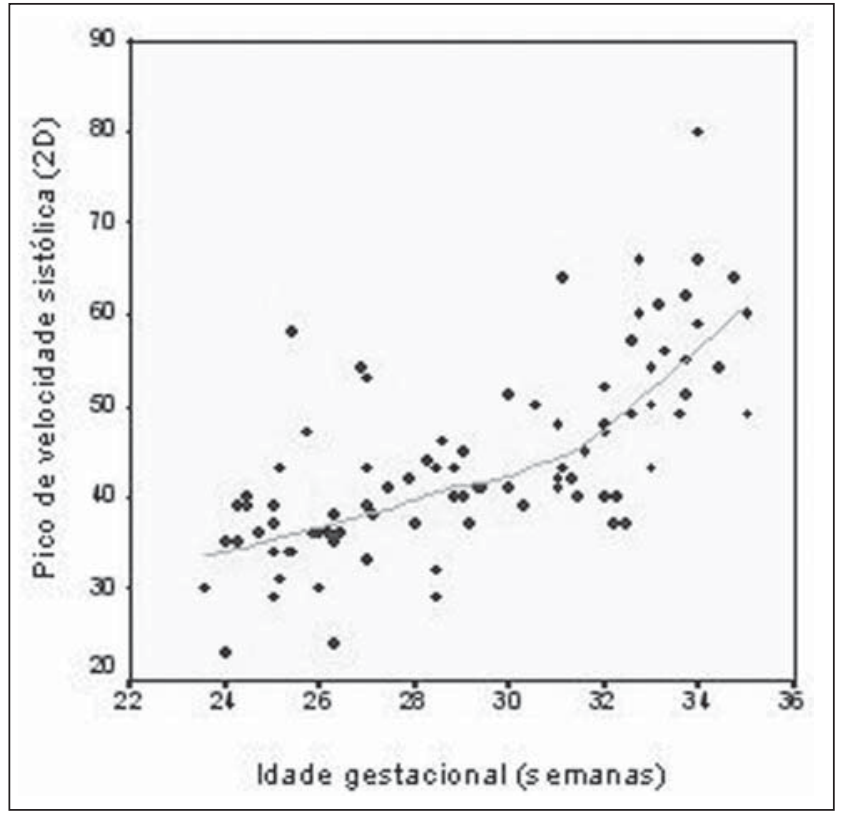

Figura 1. Correlação entre o pico de velocidade sistólica da artéria cerebral média e a idade gestacional.
Percentil $=$ média $+\mathrm{K}(\mathrm{DP})$

onde: K é o percentil correspondente à distribuição normal padrão.

A Tabela 2 demonstra o cálculo dos percentis 2,5 e 97,5 , com intervalo de $95 \%$ de confiança, utilizando $\mathrm{K}=1,96$.

A Figura 2 demonstra o comportamento da média e dos percentis 2,5 e 97,5 do PVSACM em relação à idade gestacional.

Tabela 2 Valores de referência, com intervalo de 95\% de confiança, para o pico de velocidade sis tólica da artéria cerebral média $(\mathrm{cm} / \mathrm{s})$ entre 23 e 35 semanas de gestação.

\begin{tabular}{cccc}
\hline & \multicolumn{3}{l}{ Intervalo de confiança de 95\% } \\
\cline { 2 - 4 } IG (sem.) & $\begin{array}{c}\text { PVS-ACM } \\
\text { esperado }\end{array}$ & P 2,5 & P 97,5 \\
\hline 23 & 35,44 & 24,33 & 46,55 \\
24 & 35,48 & 24,64 & 46,33 \\
25 & 35,81 & 25,08 & 46,54 \\
26 & 36,45 & 25,68 & 47,23 \\
27 & 37,43 & 26,45 & 48,41 \\
28 & 38,77 & 27,43 & 50,10 \\
29 & 40,49 & 28,63 & 52,34 \\
30 & 42,61 & 30,08 & 55,13 \\
31 & 45,16 & 31,81 & 58,51 \\
32 & 48,17 & 33,83 & 62,51 \\
33 & 51,65 & 36,16 & 67,13 \\
34 & 55,63 & 38,84 & 72,41 \\
35 & 60,13 & 41,89 & 78,36 \\
\hline
\end{tabular}

IG (sem.), idade gestacional em semanas; PVS-ACM pico de velocidade sistólica na artéria cerebral média; $\mathrm{P}$, percentil. 


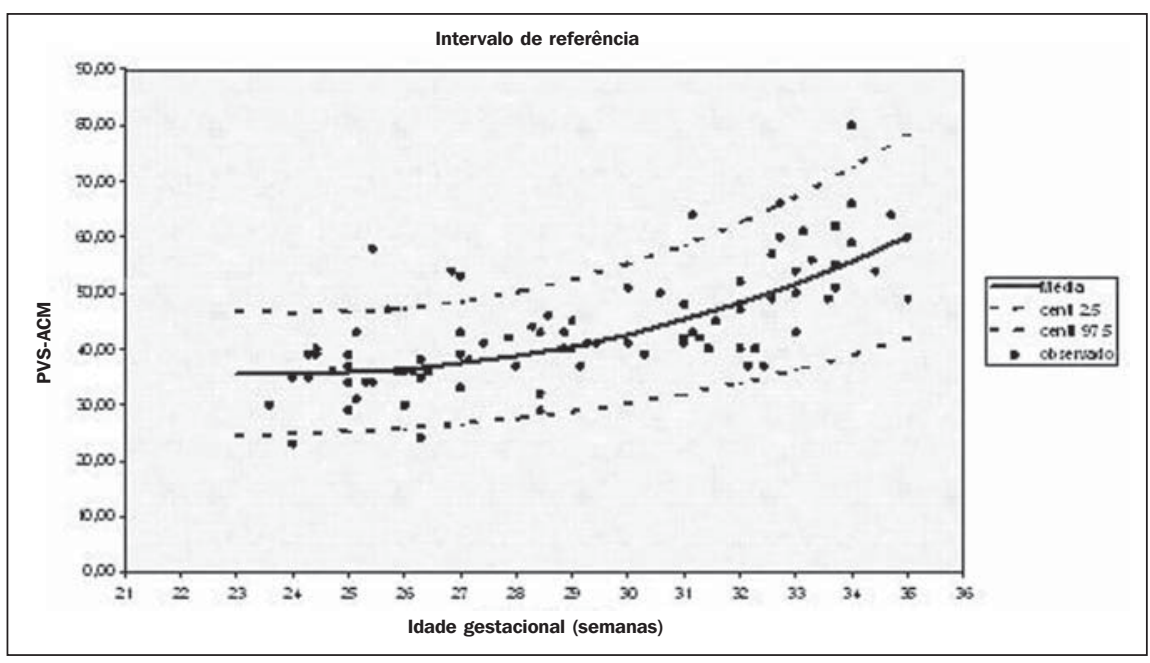

Figura 2. Comportamento do pico de velocidade sistólica na artéria cerebral média (média, percentil $2,5$ e percentil 97,5$)$ em relação à idade gestacional.

\section{DISCUSSÃO}

A aloimunização de células vermelhas do sangue continua a ser grave complicação da gestação, a despeito de sua fisiopatologia já ter sido reconhecida e o seu tratamento, estabelecido. Em países em desenvolvimento, a incidência desta doença tem decrescido muito vagarosamente, principalmente por causa da deficiente profilaxia pré-natal com anti-D gamaglobulina.

Em estudo anterior realizado por nosso grupo, encontramos $39 \%$ de prematuridade, $31 \%$ de baixo peso ao nascimento e $10 \%$ de recém-nascidos com necessidade de transfusão em relação ao grupo controle ${ }^{(10)}$. Portanto, a detecção precoce da anemia hemolítica fetal, com rápida intervenção por meio de transfusão intra-uterina ou antecipação do parto para terapêutica neonatal, pode-se acompanhar de bons resultados neonatais ${ }^{(\mathbf{1 1 1})}$.

Os métodos invasivos existentes para quantificar o grau de anemia fetal são a amniocentese e a cordocentese. A amniocentese apresenta resultado questionável quando realizada abaixo de 27 semanas $^{(\mathbf{1 2})}$; além do mais, apresenta complicações como hemorragia feto-materna, que pode piorar a gravidade da doença ${ }^{(13)}$, e a necessidade de amniocenteses seriadas ${ }^{(14)}$. Já a cordocentese apresenta maior risco de perda fetal em relação à amniocentese, sendo que a punção transplacentária está associada com hemorragia feto-materna e aumento da sensibilização ${ }^{(15)}$.
Os métodos não-invasivos de quantificação do grau de anemia fetal apresentam como grande benefício a ausência dos riscos materno-fetais existentes com os métodos invasivos.

No final da década de 80 , diversos autores avaliaram diferentes vasos fetais com o modo Doppler, na tentativa de obterem um método não-invasivo de quantificação do grau de anemia fetal nos casos de aloimunização de células vermelhas do sangue, entretanto, não obtiveram boa correlação entre esses parâmetros ${ }^{(5,16)}$.

Em 1995, Mari et al..$^{(7)}$ demonstraram que a velocidade de fluxo sanguíneo em fetos anêmicos está aumentada em relação aos fetos normais. Esses autores utilizaram a ACM, porque este vaso é facilmente identificado e seu fluxo é estudado com um ângulo baixo e diferente de $0^{\circ}$. O PVS-ACM pode ser medido acuradamente, com baixa variabilidade intra- e interobservador. Posteriormente, vários estudos comprovaram a superioridade da medida do PVS-ACM sobre a espectrofotometria do líquido amniótico na predição de anemia fetal ${ }^{(11,17-20)}$. Em um estudo realizado por nosso grupo, comprovamos que os recém-nascidos de gestantes Rh-aloimunizadas seguidas com medida do PVS-ACM tiveram maior hematócrito e menor necessidade de transfusão neonatal do que as gestantes seguidas com amniocenteses ${ }^{(21)}$.

Em nosso estudo observamos forte correlação entre o PVS-ACM e a idade gestacional, sendo que o melhor modelo foi uma exponencial. Esse modelo exponencial está de acordo com a curva de referência de 135 gestantes no estudo pioneiro realizado por Mari et al. ${ }^{(7)}$. Esse mesmo modelo exponencial foi obtido por Alshimmiri et al. ${ }^{(22)}$ com 300 fetos normais. Entretanto, Gadelha da Costa et al. ${ }^{(23)}$ obtiveram uma relação linear entre o PVS-ACM e a idade gestacional: PVS-ACM: 21,47 + 2,16GA.

Um objetivo do presente estudo foi determinar uma curva de referência para o PVS-ACM usando múltiplos da mediana segundo proposto por Mari et al. ${ }^{(8)}$. Determinamos valores para os seguintes múltiplos da mediana: 1,$0 ; 1,29 ; 1,50 ; 1,55$. O valor de 1,29 múltiplo da mediana representa o cut-off para a detecção de anemia leve, o valor de 1,50 o cut-off para a detecção de anemia moderada e o valor de 1,55 o cut-off para a deteç̧ão de anemia acentuada, segundo proposto por Mari et al. ${ }^{(8)}$. No estudo de Mari et al. ${ }^{(8)}$, os autores obtiveram sensibilidade da $100 \%$, com taxa de falso-positivo de $12 \%$ para o aumento do PVS-ACM na predição de anemia fetal moderada ou grave. Comparando a nossa curva com a de Mari et al. ${ }^{(8)}$, notamos que nossos valores são maiores em todas as idades gestacionais. Entre os motivos desta diferença poderíamos citar a diferença étnica entre as duas populações, o grau nutricional das gestantes envolvidas e a altitude dos locais onde foram realizadas as pesquisas, fatores estes que justificariam a determinação de uma curva de normalidade para diferentes populações.

Um outro objetivo do presente estudo foi determinar valores de referência baseados em percentis para o PVS-ACM. Observamos aumento progressivo da PSV-ACM na segunda metade da gestação, da mesma forma que o obtido por Gadelha da Costa et al. ${ }^{(23)}$, Kurmanavicius et al. ${ }^{(24)}$ e Bahlmann et al. ${ }^{(25)}$. Em comparação ao estudo de Gadelha da Costa et al. ${ }^{(23)}$, que também utilizaram uma amostra da população brasileira, observamos valores semelhantes do PVS-ACM no período de 22 a 34 semanas. Em comparação ao estudo de Kurmanavicius et al. ${ }^{(24)}$, que também utilizaram os critérios de Royston e Wright ${ }^{(9)}$ para a determinação dos percentis do PVS-ACM, observamos valores semelhantes no período gestacional estudado, entretanto, esses autores obtiveram correlação linear entre os 
valores do PVS-ACM e a idade gestacional, enquanto em nosso estudo obtivemos uma equação cúbica. Em comparação ao estudo de Bahlmann et al. ${ }^{\text {(25) }}$, os nossos valores do PVS-ACM foram maiores para um mesmo intervalo gestacional (23 a 35 semanas), e uma possível explicação poderia ser o método aplicado naquele estudo, que utilizou intervalo de $90 \%$ de confiança, enquanto em nosso estudo utilizamos intervalo de $95 \%$ de confiança.

Em síntese, acreditamos que este estudo tenha sido importante, pois é o segundo estudo a determinar uma curva de referência para o PVS-ACM utilizando múltiplos da mediana. $\mathrm{O}$ fato de este estudo piloto demonstrar que nossos valores são diferentes dos obtidos por Mari et al. ${ }^{(\mathbf{8})}$ justifica um estudo mais amplo na população brasileira, de preferência multicêntrico, para se comprovar realmente se essa discordância se deve a fatores étnicos. De qualquer forma, acreditamos que este estudo tenha sido um primeiro passo na determinação de uma curva de referência para o PVS-ACM utilizando múltiplos da mediana, e que futuramente possa servir para o seguimento de gestantes com aloimunização de células vermelhas.

\section{REFERÊNCIAS}

1. Liley AW. Liquor amnii analysis in the management of the pregnancy complicated by rhesus sensitization. Am J Obstet Gynecol. 1961;82:135970 .

2. Simpson JL. Incidence and timing of pregnancy losses: relevance to evaluating safety of early prenatal diagnosis. Am J Med Genet. 1990;35:16573

3. MacKenzie IZ, Bowell PJ, Castle BM, et al. Serial fetal blood sampling for the management of pregnancies complicated by severe rhesus (D) isoimmunization. Br J Obstet Gynaecol. 1988;95: 753-8.

4. Nicolaides KH, Soothill PW, Clewell WH, et al. Fetal haemoglobin measurement in the assessment of red cell isoimmunisation. Lancet. 1988;1: 1073-5.

5. Nicolaides KH, Bilardo CM, Campbell S. Prediction of fetal anemia by measurement of the mean blood velocity in the fetal aorta. Am J Obstet Gynecol. 1990;162:209-12.

6. Copel JA, Grannum PA, Green JJ, et al. Pulsed Doppler flow-velocity waveforms in the prediction of fetal hematocrit of the severely isoimmunized pregnancy. Am J Obstet Gynecol. 1989; 161:341-4.

7. Mari G, Adrignolo A, Abuhamad AZ, et al. Diagnosis of fetal anemia with Doppler ultrasound in the pregnancy complicated by maternal blood group immunization. Ultrasound Obstet Gynecol. 1995;5:400-5.

8. Mari G, Deter RL, Carpenter RL, et al. Noninvasive diagnosis by Doppler ultrasonography of fetal anemia due to maternal red-cell alloimmunization. N Engl J Med. 2000;342:9-14.

9. Royston P, Wright EM. How to construct "normal ranges' for fetal variables. Ultrasound Obstet Gynecol. 1998;11:30-8.

10. Nardozza LM, Camano L, Moron AF, et al. Pregnancy outcome for $\mathrm{Rh}$-alloimmunized women. Int J Gynaecol Obstet. 2005;90:103-6.

11. Oepkes D. Invasive versus non-invasive testing in red-cell alloimunized pregnancies. Eur J Obstet Gynecol Reprod Biol. 2000;92:83-9.

12. Rahman F, Detti L, Ozcan T, et al. Can a single measurement of amniotic fluid delta optical density be safely used in the clinical management of Rhesus-alloimmunized pregnancies before 27 weeks' gestation? Acta Obstet Gynecol Scand. 1998;77:804-7.

13. Bowman JM, Pollock JM. Transplacental fetal hemorrhage after amniocentesis. Obstet Gynecol. 1985;66:749-54.

14. Queenan JT, Tomai TP, Ural SH, et al. Deviation in amniotic fluid optical density at a wavelength of $450 \mathrm{~nm}$ in Rh-immunized pregnancies from 14 to 40 weeks' gestation: a proposal for clinical management. Am J Obstet Gynecol. 1993;168: 1370-6.
15. MacGregor SN, Silver RK, Sholl JS. Enhanced sensitization after cordocentesis in a rhesusisoimmunized pregnancy. Am J Obstet Gynecol. 1991;165:382-3

16. Rightmire DA, Nicolaides $\mathrm{KH}$, Rodeck $\mathrm{CH}$, et al. Fetal blood velocities in Rh isoimmunization: relationship to gestational age and to fetal hematocrit. Obstet Gynecol. 1986;68:233-6.

17. Nishie EN, Brizot ML, Liao AW, et al. A comparison between middle cerebral artery peak systolic velocity and amniotic fluid optical density at 450 $\mathrm{nm}$ in the prediction of fetal anemia. Am J Obstet Gynecol. 2003;188:214-9.

18. Oepkes D, Seaward PG, Vandenbussche FP, et al. Doppler ultrasonography versus amniocentesis to predict fetal anemia. N Engl Med J. 2006;355: 156-64.

19. Pastore AR. Dopplervelocimetria da artéria cerebral média fetal: o divisor de águas no diagnóstico da anemia fetal. Radiol Bras. 2006;39(1):iii.

20. Nardozza MM, Camano L, Moron AF, et al. Alterações ultra-sonográficas na gravidez $\mathrm{Rh}$ negativo sensibilizada avaliada pela espectrofotometria do líquido amniótico e pela dopplervelocimetria da artéria cerebral média. Radiol Bras. 2006; 39:11-3.

21. Nardozza LM, Moron AF, Araujo Júnior E, et al. $\mathrm{Rh}$ alloimmunization: Doppler or amniotic fluid analysis in the prediction of fetal anemia? Arch Gynecol Obstet. 2007;275:107-11.

22. Alshimmiri MM, Hamoud MS, Al-Saleh EA, et al. Prediction of fetal anemia by middle cerebral artery peak systolic velocity in pregnancies complicated by Rhesus isoimmunization. J Perinatol. 2003;23:536-40

23. Gadelha Da Costa A, Mauad Filho F, Spara P, et al. Fetal hemodynamics evaluated by Doppler velocimetry in the second half of pregnancy. U1trasound Med Biol. 2005;31:1023-30.

24. Kurmanavicius J, Streicher A, Wright EM, et al Reference values of fetal peak systolic blood flow velocity in the middle cerebral artery at 19-40 weeks of gestation. Ultrasound Obstet Gynecol. 2001;17:50-3.

25. Bahlmann F, Reinhard I, Krummenauer F, et al. Blood flow velocity waveforms of the fetal middle cerebral artery in a normal population: reference values from 18 weeks to 42 weeks of gestation. J Perinat Med. 2002;30:490-501. 